\title{
Inverse Measurement of Moisture Content in Porous Insulation Materials with a Data Sorting Method
}

\author{
Huojun Yang ${ }^{1}$, Yun Luo ${ }^{2}$ and Tengfei (Tim) Zhang ${ }^{2}$ \\ 1. Construction Management and Engineering Department, North Dakota State University, ND 58108, USA \\ 2. School of Civil Engineering, Dalian University of Technology, Liaoning 116024, China
}

Received: July 08, 2016 / Accepted: July 22, 2016 / Published: November 30, 2016.

\begin{abstract}
Moisture in insulation materials will impair their thermal and acoustic performance, induce microbe growth, and cause equipment/material corrosion. Moisture content measurement is vital to the effective moisture control. This investigation proposes a simple, fast, and accurate method to measure moisture content of insulation materials through matching the measured temperature rise. Since moisture content corresponds to unique thermophysical properties, the measured temperature rise varies with moisture content. During the data analysis, all possible volumetric heat capacities and thermal conductivities are enumerated to match the measured temperature rise based on the composite heat conduction theory. Then, the partial derivatives with respect to both volumetric heat capacity and thermal conductivity are evaluated, so that these partial derivatives will be guaranteed equaling to zero at the optimal solutions to the moisture content. Compared to the benchmarked gravimetric method, this proposed method was found having a better accuracy but requiring a short test time.
\end{abstract}

Key words: Moisture content, data sort, temperature match, composite heat conduction.

\section{Introduction}

Moisture content in porous materials exerts great impacts to their thermal insulation, acoustics, processability, dielectricity, storage life, and chemical aspects, etc. Moisture content, simply "the quantity of moisture in a material", can be defined by "volume or mass" basis and "wet or dry" basis. In this study, wet basis is used, including wet basis volumetric moisture content $\theta_{\mathrm{w}}$ (volume of water divided by the bulk volume of wet material) and wet basis mass moisture content $y$ (mass of water divided by the bulk mass of wet material).

Moisture content can be either directly or indirectly determined. The direct techniques measure moisture by a chemical reaction (i.e., the Karl Fisher titration method) or weighing the mass difference before and after drying a test sample. The latter one is usually

Corresponding author: Tengfei (Tim) Zhang, Ph.D., professor, research field: built environment. referred as the LOD (loss on drying) or as the thermo-gravimetric method (or simply gravimetric). Although the direct methods are accurate, they are time-consuming, destructive, and do not allow for the in-situ measurement. Therefore, a lot of indirect methods have been alternatively developed. Unlike the direct measurements, indirect techniques do not involve the removal of moisture in a sample, while they estimate moisture content by a strong or calibrated relationship with some other measurable variables such as dielectric permittivity or thermal conductivity. Both the empirical and theoretical equations between the moisture content and measurable variable are used for the calibration. In terms of working principles, these indirect methods are mainly classified as neutron moderation, gamma ray attenuation, nuclear magnetic resonance, microwave reflectance and attenuation, NIR/T (near infrared reflectance or transmission), dielectric techniques, thermal methods, etc.

The suitability of a measurement method relies on 
many issues, such as safety, cost, accuracy, response time, installation, ease of operation, management, and durability. Thermal techniques are promisingly considered as an alternative to other methodologies to determine moisture content in porous materials. One typical example identifies moisture content $\theta_{v}$ by its relationship with volumetric specific heat capacity ( $\rho c$ ) [1-4]. The main attractions in pursuing this technology are: simultaneous measurements of other thermophysical properties (e.g., thermal conductivity, thermal diffusivity, volumetric heat capacity, and water matric potential), wide measurement range, invulnerability of salinity, low cost, high accuracy and robustness, etc.

In this study, we will continue to follow our previous research using the DP (dual-probe) thermal technique, where one probe contains a line-source heater and the other (separated in parallel by a distance) contains a temperature sensor. The typical DP thermal technique uses dual-probe heat-pulse probe $[3,5]$ where a transient heat-pulse is simulated by powering a heater for a few seconds, and the sensor measures the temperature response during the next several minutes. However, this simulation of transient heat-pulse through a short time heating will bring an error to this measurement model, and the transient heating may only allow for small volume sensing because temperature response will not be sufficient for a big distance between the two probes. The long measurement time (several minutes) may not be acceptable for some applications.

Recently, Zhang et al. [6] used a sudden constant heating, instead of heat-pulse, in a cylindrical heat source, where a uniform and constant heating was provided during the whole process of measurement. Compared to the heat-pulse technique, this method has heating for a longer time and in turn provides enough temperature response which can be sensed by a sensor further away from the heat source, and then a bigger volume sensing is allowed. The results from Zhang et al. [6] showed that this method has a very high accuracy but the measurement time still needs to be shortened. Thus, this paper, by matching the measured temperature response profile with the predicted one, attempts to reduce the total measurement time without sacrificing the high accuracy.

\section{Methods}

\subsection{Basic Equations of Moisture Content in Porous Materials}

When containing moisture, a porous medium can be considered as a combination of three components: air, water, and solid (the medium matrix). Like the mass additive law, the additive law also applies to the volumetric heat capacity (density multiplying with specific heat), which is expressed as:

$$
(\rho c)_{\mathrm{w}} \theta_{\mathrm{w}}+(\rho c)_{\mathrm{s}} \theta_{\mathrm{s}}+(\rho c)_{\text {air }} \theta_{\text {air }}=(\rho c)_{\mathrm{wet}}
$$

where, $\rho$ is density, $c$ is specific heat, $\theta$ is volumetric fraction, and subscripts $w, s$, air and wet refer to the water, solid skeleton, air and wet porous medium, respectively.

In Eq. (1), the contribution of gaseous substances to the overall volumetric heat capacity can be omitted due to relatively small density and specific heat of common gases. Such approximation is suitable because, taking the air as an example, the air density is smaller than the water density by three magnitudes of order, in addition to its smaller specific heat. Eq. (1) can also be rewritten into:

$$
(\rho c)_{\mathrm{w}} \theta_{\mathrm{w}}+(\rho c)_{\mathrm{dry}}=(\rho c)_{\mathrm{wet}}
$$

where, the subscript dry refers to the composite of the solid porous media matrix and air. Furthermore, as the volumetric heat capacity is defined as the ratio of the thermal conductivity with the thermal diffusivity, Eq. (2) can be rearranged into:

$$
(\rho c)_{\mathrm{w}} \theta_{\mathrm{w}}+\left(\frac{k}{a}\right)_{\mathrm{dry}}=\left(\frac{k}{a}\right)_{\mathrm{wet}}
$$

where, $k$ and $a$ refer to the thermal conductivity and the thermal diffusivity, respectively. For each specific water content, the wet porous material has unique $k$ and $a$. 
After the volumetric fraction of water is obtained, its mass fraction of water $y_{\mathrm{w}}$ (wet basis) can accordingly be determined by

$$
y_{\mathrm{w}}=\theta_{\mathrm{w}} \cdot \frac{\rho_{\mathrm{w}}}{\rho_{\mathrm{wet}}}=\frac{\theta_{\mathrm{w}} \cdot \rho_{\mathrm{w}}}{\theta_{\mathrm{w}} \cdot \rho_{\mathrm{w}}+\rho_{\mathrm{dry}}}
$$

\subsection{Composite Heat Conduction Subject to a Sudden} Constant Heating by an Infinite Cylindrical Source

Suppose that the initial temperature distribution in the test medium is uniform, and the cylindrical heat element and the sensor are in good contact with the porous test medium. Considering the finite heat capacity of the heat element and its finite diameter, according to Refs. [7, 8], the analytical solution to the temperature rise due to a sudden constant heating by an infinite cylindrical source is

$$
\begin{aligned}
& \Delta T_{\mathrm{E}}(r, t)=\frac{4 q k a}{\pi^{3} r_{0}^{3}} \int_{0}^{\infty} \frac{\left(1-e^{-a_{\mathrm{E}} u^{2} t}\right) J_{0}(u r) J_{1}\left(u r_{0}\right)}{u^{4}\left[\phi^{2}(u)+\psi^{2}(u)\right]} d u \\
& \text { for } \quad r<r_{0}
\end{aligned}
$$

and

$$
\begin{aligned}
& \Delta T(r, t)=\frac{2 q a^{1 / 2}}{\pi^{2} r_{0}^{2}} \int_{0}^{\infty}\left(1-e^{-a_{E} u^{2} t}\right) J_{1}\left(u r_{0}\right) \times \\
& {\left[J_{0}\left(\sqrt{a_{E} / a} u r\right) \phi(u)-Y_{0}\left(\sqrt{a_{E} / a} u r\right) \psi(u)\right]} \\
& \times\left\{u^{3}\left[\phi^{2}(u)+\psi^{2}(u)\right]\right\}^{-1} d u
\end{aligned}
$$

for

$$
r>r_{0}
$$

where, $\Delta T$ is temperature rise with respect to the initial status, $t$ is heating time, $r$ is the distance from the center of cylindrical heating element, $r_{0}$ is the radius of the heating element, $q$ is the heat generation rate per unit length, the subscript $E$ represents the heat element, $J_{0}$ and $Y_{0}$ are the first and the second kind Bessel function of the zero order, respectively, $J_{1}$ and $Y_{1}$ are the first and second kind Bessel function of the first order, respectively, $\phi(u)$ and $\psi(u)$ are functions of the above variables and Bessel functions.

Eq. (6) provides a unique transient temperature response profile for a set of $(k, a)$ which is subject to the moisture content. In this study, various sets of $(k, a)$ are enumerated to predict a temperature response profile based on Eq. (6). If the predicted temperature response profile closely matches with the measured one, the enumerated set of $(k, a)$ is considered as actual thermal properties and then the moisture content is determined by Eqs. (3) and (4). Although $k$ or $a$ alone is highly influenced by the contact resistance, their ratio, the volumetric heat capacity $\rho c$, is immune to the contact resistance. This is because the volumetric heat capacity of the air is much smaller than that of the solid skeleton of the porous medium or the water.

\subsection{Temperature Response Matching Methods}

According to Eq. (6), $\Delta T(r, t)$ is a function of independent variables of $t$ (heating time), $r$ (distance from the center of cylindrical heating element to temperature sensor), $r_{0}$ (radius of the heating element), $q$ (heat generation rate per unit length), $\left(k_{\text {wet }}, a_{\text {wet }}\right)$ (thermal conductivity and diffusivity of wet porous medium, respectively, and $\left(k_{\mathrm{E}}, a_{\mathrm{E}}\right)$ (thermal conductivity and thermal diffusivity of heat element, respectively). For a specific set of experiments, $r, r_{0}, q$, and $\left(k_{\mathrm{E}}, a_{\mathrm{E}}\right)$ are known, so $\Delta T(r, t)$ can be expressed into a function of ( $\left.k_{\mathrm{wet}}, a_{\mathrm{wet}}\right)$ :

$$
\Delta T(t)=f\left(t, k_{\mathrm{wet}}, a_{\mathrm{wet}}\right)
$$

Therefore, each recorded or measured temperature response $\Delta T_{\mathrm{M}, i}$ corresponds to its predicted temperature response $\Delta T_{\mathrm{P}, i}$ according to Eq. (7), where, subscripts $\mathrm{M}, \mathrm{P}, i$ are measured, predicted, and the $i$ th time step of a test, respectively. Then, as shown in Eq. (8), the root-mean-square temperature difference $D$ between $\Delta T_{\mathrm{M}, i}$ and $\Delta T_{\mathrm{P}, i}$ can be used to quantify the matching degree between the measurement and the prediction as:

$$
D=\sqrt{\sum_{i=1}^{n}\left(\Delta T_{\mathrm{P}, i}-\Delta T_{\mathrm{M}, i}\right)^{2} / n}=f\left[k_{\mathrm{wet}},(\rho c)_{\mathrm{wet}}\right](8)
$$

It can be seen that $D$ is a function of ( $\left.k_{\text {wet }}, a_{\text {wet }}\right)$ and also one of $\left[k_{\text {wet }},(\rho c)_{\text {wet }}\right]$. For illustration, Fig. 1 
shows two situations, where the $D$ value of Fig. 1a is larger than that of Fig. 1b, and thus Fig. $1 \mathrm{~b}$ has a better temperature match than Fig. 1a.

Theoretically, a measured temperature response with time corresponds to a unique $\left[k_{\text {wet }},(\rho c)_{\text {wet }}\right]$ that makes the temperature difference $D$ equal to zero. That is, if a set of $\left[k_{\text {wet }},(\rho c)_{\text {wet }}\right]$ is found to have $D=0$, then it is the actual thermal conductivity and diffusivity of the tested porous material. The problem of solving $D=f\left[k_{\text {wet }},(\rho c)_{\text {wet }}\right]=0$ can be resorted to the optimal solution problem in the Calculus. If there is one point, say $\left[k_{\mathrm{wet}}^{0},(\rho c)_{\mathrm{wet}}^{0}\right]$, whose partial derivatives $\partial D / \partial k_{\text {wet }}$ and $\partial D / \partial(\rho c)_{\text {wet }}$ are both equal to zero, then the function $D=0$ has the only optimal solution at the point $\left[k_{\text {wet }}^{0},(\rho c)_{\text {wet }}^{0}\right]$. In brief, the measured moisture content can be determined by solving equations:

$$
\partial D / \partial k_{\text {wet }}=0 \text { and } \partial D / \partial(\rho c)_{\text {wet }}=0
$$

According to the definition of extreme points, any point satisfying $\partial D / \partial k_{\text {wet }}=0$ at a fixed $(\rho c)_{\text {wet }}$ is an extreme point. Similarly, any point satisfying $\partial D / \partial(\rho c)_{\text {wet }}=0$ at a fixed $k_{\text {wet }}$ is also an extreme



(a) point. The optimal solution $\left[k_{\mathrm{wet}}^{0},(\rho c)_{\mathrm{wet}}^{0}\right]$ comes from these extreme points. However, it is very difficult to obtain a theoretical solution for the above equations. Fortunately, both $\partial D / \partial k_{\text {wet }}$ and $\partial D / \partial(\rho c)_{\text {wet }}$ have a limited number of extreme points in this study's application range. As shown in Fig. 2, there is only one extreme point for $\partial D / \partial(\rho c)_{\text {wet }}$ at each fixed $k_{\text {wet }}$, and $D$ varies with $(\rho c)_{\text {wet }}$ like a parabola.

Due to various error sources such as the sensor's sensitivity and accuracy, there are a set of optimal points rather than the only one. In this study, all the optimal points are considered effective to assess the moisture content, and the final result of measured moisture content can be expressed as the range, average, median, or minimum of all these moisture contents calculated from these optimal points.

The data sort procedure is as follows: enumerating all possible $\left[k_{\text {wet }},(\rho c)_{\text {wet }}\right]$ sets; obtaining data series for both $\partial D / \partial k_{\text {wet }}$ and $\partial D / \partial(\rho c)_{\text {wet }}$ for each $\left[k_{\text {wet }},(\rho c)_{\text {wet }}\right]$; assuming the $\left[k_{\text {wet }},(\rho c)_{\text {wet }}\right]$ as an extreme point when its $D$ value is minimal in a continuous range; and taking the extreme point as an optimal point when both $\partial D / \partial k_{\text {wet }}=0$ and $\partial D / \partial(\rho c)_{\mathrm{wet}}=0$.

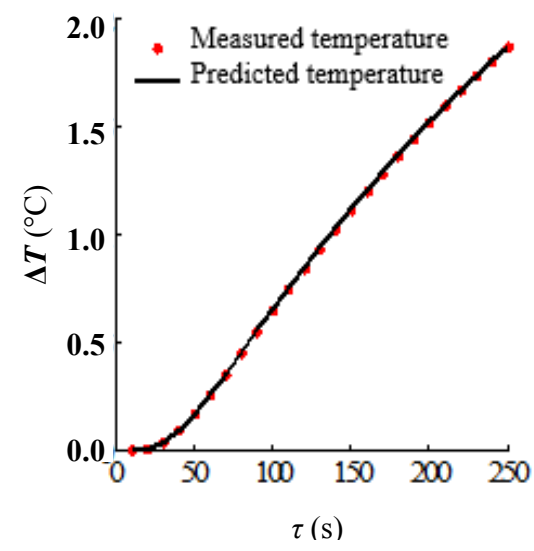

(b)

Fig. 1 Matching of temperature rise between measurement and prediction: (a) an inferior match with a large $D$; (b) a superior match with a small $D$. 


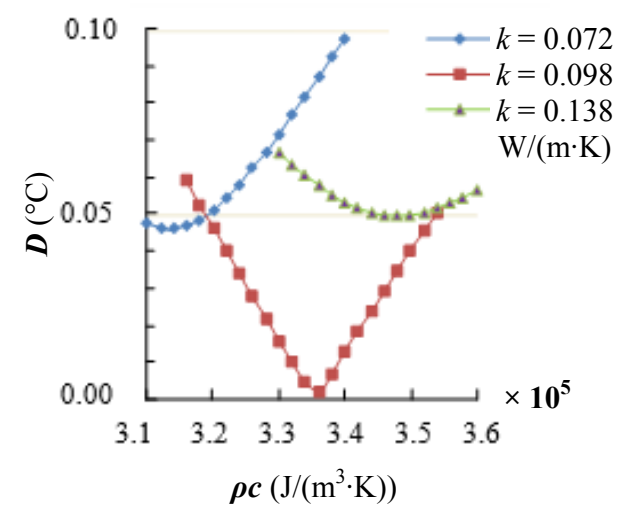

Fig. 2 Variation of $D$ with $(\rho c)_{\text {wet }}$ at different fixed $k_{\text {wet }}$ values for a constant moisture content.

\section{Results}

A nichrome wire was used as the cylindrical heat source for moisture content measurement in a sponge block. Fig. 3 shows the test facility. The thin hot wire goes through the test medium which is mounted in a box. A Pt100 temperature sensor probe is fixed at one side of the box, separated in parallel from the hot wire by a distance of $11 \mathrm{~mm}$. The length of the temperature probe is $42 \mathrm{~mm}$, and its diameter is $5 \mathrm{~mm}$. The resolution of temperature measurement is $0.1{ }^{\circ} \mathrm{C}$ with an accuracy of $\pm(0.30+0.005|T|){ }^{\circ} \mathrm{C}$. The hot wire is powered by a DC voltage source. In addition, a digital precision balance (type: SI-6002; Denver Instrument, USA) was adopted to weight the moisture content as a benchmark. The resolution of the digital balance is 0.01 $\mathrm{g}$ with an accuracy of $\pm 0.02 \mathrm{~g}$.

Table 1 shows the experiment results of four cases, including $y_{\mathrm{G}}$ (mass moisture content with the gravimetric method), the search range and step size for both $k_{\text {wet }}$ and $(\rho c)_{\text {wet }}$ when they are enumerated during the match of measured temperature rise, and the indicated mass moisture content $y_{\mathrm{M}}$ using the proposed method.

For each case, 40 data points of temperature rise response were measured for totally $200 \mathrm{~s}$, i.e., $n=40$ in the Eq. (8). Let us take Case 4 as an example to illustrate the results. According to an estimation to thermophysical properties for the mixed materials, the potential $k_{\text {wet }}$ and $(\rho c)_{\text {wet }}$ of the tested material are within the ranges of $0.04 \sim 0.18 \mathrm{~W} /(\mathrm{m} \cdot \mathrm{K})$ and $3.2 \sim 4.9 \times$ $10^{5} \mathrm{~J} /\left(\mathrm{m}^{3} \cdot \mathrm{K}\right)$, respectively. The changing step size is set to be $0.002 \mathrm{~W} /(\mathrm{m} \cdot \mathrm{K})$ and $0.05 \times 10^{5} \mathrm{~J} /\left(\mathrm{m}^{3} \cdot \mathrm{K}\right)$ accordingly. Hence, each $(\rho c)_{\text {wet }}$ corresponds to 70 different $k_{\text {wet }} \mathrm{s}$, and there are $70 \partial D / \partial k_{\text {wet }} \mathrm{s}$. Similarly, each $k_{\text {wet }}$ corresponds to $34(\rho c)_{\text {wet }}$ s and there are $\partial D / \partial(\rho c)_{\text {wet }}$ s. In total, there are 2,380 (i.e., $70 \times 34)$ combinations of $\left[k_{\mathrm{wet}},(\rho c)_{\mathrm{wet}}\right]$ for this case.

Next, the $D$ value is calculated for each of the 2,380 combinations according to Eqs. (6) and (8). Then, these data are initially screened by $\left(D<0.5{ }^{\circ} \mathrm{C}\right)$ and ( $a_{\text {moisture }}<a_{\text {wet }}<a_{\text {air }}$ ) where the thermal diffusivity $a_{\text {wet }}=k_{\text {wet }} /(\rho c)_{\text {wet }}$, and there are 279 combinations satisfying the above constraints, as shown in Table 2.

Then extreme points are determined for each $\partial D / \partial k_{\text {wet }}$ and $\partial D / \partial(\rho c)_{\text {wet }}$. In order to avoid removing true optimal solutions, an error threshold $0.005{ }^{\circ} \mathrm{C}$ is imposed to $D$ during evaluating the partial

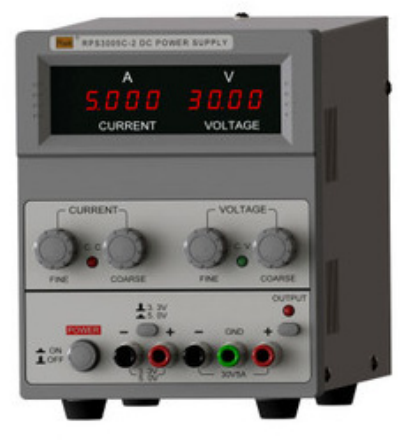

(b)

Fig. 3 Test facility: (a) test device including a hot wire and a Pt 100 temperature sensor; (b) a DC voltage supplier. 
Table 1 Experiment results for the hot wire test.

\begin{tabular}{lllllll}
\hline No. & $y_{\mathrm{G}}$ & Range of $k_{\text {wet }}$ & Step size of $k_{\text {wet }}$ & Range of $(\rho c)_{\text {wet }}$ & Step size of $(\rho c)_{\text {wet }} y_{\mathrm{M}}$ \\
\cline { 2 - 7 } & $\mathrm{g} / \mathrm{g}$ & $\mathrm{W} /(\mathrm{m} \cdot \mathrm{K})$ & $\mathrm{W} /(\mathrm{m} \cdot \mathrm{K})$ & $10^{5} \mathrm{~J} /\left(\mathrm{m}^{3} \cdot \mathrm{K}\right)$ & $10^{5} \mathrm{~J} /\left(\mathrm{m}^{3} \cdot \mathrm{K}\right)$ & $\mathrm{g} / \mathrm{g}$ \\
\hline 1 & 0 & $0.030 \sim 0.060$ & 0.001 & $1.20 \sim 1.80$ & 0.01 & $\mathrm{~N} / \mathrm{A}$ \\
2 & $50.60 \%$ & $0.040 \sim 0.120$ & 0.002 & $2.20 \sim 3.20$ & 0.02 & $51.70 \sim 52.20 \%$ \\
3 & $66.25 \%$ & $0.040 \sim 0.150$ & 0.002 & $2.90 \sim 3.80$ & 0.02 & $65.03 \% \sim 66.03 \%$ \\
4 & $74.43 \%$ & $0.040 \sim 0.180$ & 0.002 & $3.20 \sim 4.90$ & 0.05 & $73.04 \% \sim 74.36 \%$ \\
\hline
\end{tabular}

Table 2 The snapshot of potential combinations of thermal conductivity and volumetric heat capacity after the initial screen.

\begin{tabular}{llll}
\hline No. & $k_{\text {wet }}$ & $(\rho c)_{\text {wet }}$ & $D\left({ }^{\circ} \mathrm{C}\right)$ \\
\hline.. &.. &.. &.. \\
27 & 0.08 & 4.9 & 0.2492 \\
28 & 0.082 & 4.0 & 0.06916 \\
.. &.. &.. &.. \\
221 & 0.116 & 4.85 & 0.0234 \\
.. &.. &.. &.. \\
\hline
\end{tabular}

Table 3 Extreme points for $\partial D / \partial k_{\text {wet }}$ and $\partial D / \partial \rho c_{\text {wet }}$.

\begin{tabular}{|c|c|c|c|c|c|c|}
\hline No. & $k_{\text {wet }}$ & $(\rho c)_{\text {wet }}$ & $D$ & $k_{\text {wet }}$ & $(\rho c)_{\text {wet }}$ & $D$ \\
\hline.. & .. & .. & .. & .. & .. & .. \\
\hline 19 & 0.08 & 4.1 & 0.29 & 0.08 & 4.1 & 0.29 \\
\hline.. & .. & .. &.. & .. & .. & .. \\
\hline 100 & 0.096 & 4.5 & 0.0117 & 0.096 & 4.5 & 0.0117 \\
\hline .. & .. & .. & .. & .. & .. & .. \\
\hline 111 & 0.098 & 4.55 & 0.0128 & 0.098 & 4.55 & 0.0128 \\
\hline .. & .. & .. & .. & .. & .. & .. \\
\hline
\end{tabular}

derivatives, i.e., if the $D$ value between an extreme point and its adjacent data point is within $[-0.005$, $0.005]$, then the adjacent data point is also considered as an extreme point. We identified 25 extreme points for $\partial D / \partial k_{\text {wet }}$ (highlighted in green in column $k_{\text {wet }}$ ) and 22 for $\partial D / \partial(\rho c)_{\text {wet }}$ (in purple in column $\left.(\rho c)_{\text {wet }}\right)$, as shown in Table 3 . If a set of $\left(k_{\text {wet }},(\rho c)_{\text {wet }}\right)$ is highlighted both in green and in purple, it is an optimal combination, just like No. 19 in Table 3. Finally, there are 14 optimal combinations whose volumetric and mass moisture contents and their ranges can be calculated from Eqs. (3) and (4), respectively.

As summarized in Table 1, the inferred moisture mass contents by this proposed method are in good agreement with the benchmarked gravimetric method because the $y_{M}$ error is within $\pm 2.3 \%(\mathrm{~g} / \mathrm{g})$ for all the tests. The accuracy of Case 4 is less than that of others whereas it used a bigger step size of $(\rho c)_{\text {wet }}$.

\section{Discussion}

The moisture indication accuracy can be further improved if a smaller step size for $\left[k_{\text {wet }},(\rho c)_{\text {wet }}\right]$ and a more appropriate error threshold are used. In addition, more criteria can be implemented to increase the accuracy in the screen phase for these possible combinations of thermophysical properties, e.g., the heat conductivity ranges of the mixed materials. The current application utilized temperature responses measured within 200 s. This shows the proposed method is promising to accurately indicate moisture content within a short test duration.

\section{Conclusions}

This paper presents a simple and accurate method to rapidly quantify moisture contents in porous insulation materials. Through matching the measured temperature 
rise after a sudden heating by a cylindrical heat source with the analytical solution of composite heat conduction, the volumetric heat capacity and thermal conductivity of the test material are identified, and then the moisture content is determined. A preliminary comparison of the measured moisture contents with the benchmarked gravimetric method shows that the proposed method can accurately indicate the acquired moisture contents within $200 \mathrm{~s}$ of measurement. The absolute moisture discrepancies between our method and the benchmarked gravimetric method are below $2.3 \%(\mathrm{~g} / \mathrm{g})$.

\section{References}

[1] Campbell, G. S., Calissendorff, C., and Williams, J. H. 1991. "Probe for Measuring Soil Specific Heat Using a Heat-Pulse Method." Soil Science Society of America Journal 55(1): 291-3.

[2] Bristow, K. L., Campbell, G. S., and Calissendorff, K. 1993. "Test of Heat-Pulse Probe for Measuring Changes in
Soil Water Content." Soil Science Society of America Journal, 57 (4): 930-4.

[3] Bristow, K. L., Kluitenberg, G. J., and Horton, R. 1994. "Measurement of Soil Thermal Properties with a Dual-Probe Heat-Pulse Technique." Soil Science Society of America Journal 58 (5): 1288-94.

[4] Bristow, K. L. 1998. "Measurement of Thermal Properties and Water Content of Unsaturated Sandy Soil Using Dual-Probe Heat-Pulse Probes." Agricultural and Forest Meterology 89 (2): 75-84.

[5] Welch, S. M., Kluitenberg, G. J., and Bristow, K. L. 1996. "Rapid Numerical Estimation of Soil Thermal Properties for a Broad Class of Heat Pulse Emitter Geometries." Measurement Science and Technology 7 (6): 932-8.

[6] Zhang, T., Shen, R., Lin, C. H., Yin, J., and Wang, S. 2015. "Measuring Moisture Content in a Porous Insulation Material Using a Hot Wire." Building and Environment 84 (January): 22-31.

[7] Healy, J. J., Groot, J. J. de, and Kestin, J. 1976. "The Theory of the Transient Hot-Wire Method for Measuring Thermal Conductivity." Physica B $+C 82$ (2): 392-408.

[8] Carslaw, H. S., and Jaeger, J. C. 1986. Conduction of Heat in Solids. New York : Oxford Science Publications. 